\title{
Kähler packings of projective complex manifolds
}

\section{Aeran Fleming ${ }^{1}$}

Received: 5 June 2019 / Revised: 26 February 2021 / Accepted: 26 June 2021 /

Published online: 23 August 2021

(C) The Author(s) 2021

\section{Abstract}

We show that the multipoint Seshadri constant determines the maximum possible radii of embeddings of Kähler balls and vice versa.

Keywords Multipoint Seshadri constant · Kähler packings · Nagata’s conjecture

Mathematics Subject Classification $14 \mathrm{C} 20 \cdot 32 \mathrm{Q} 15 \cdot 57 \mathrm{R} 17$

\section{Introduction}

Seshadri constants were first introduced by Demailly in [3] as a way of studying local positivity of ample line bundles at a given point of a variety. Since then, Seshadri constants have attracted substantial attention in the field of algebraic geometry and can be used to reformulate many classical ideas. A nice example of this is the famous conjecture of Nagata on plane algebraic curves, which in its original form is as follows:

Conjecture 1.1 Let $P_{1}, \ldots, P_{k}$ be points of $\mathbb{P}^{2}$ in general position and $m_{1}, \ldots, m_{k}$ be positive integers. Then for $k \geqslant 9$ any curve $C \in \mathbb{P}^{2}$ of degree d passing through each point $P_{i}$ with multiplicity $m_{i}$ must satisfy

$$
d \geqslant \frac{1}{\sqrt{k}} \sum_{i=1}^{k} m_{i}
$$

Using multipoint Seshadri constants this can be rewritten as:

The author was supported by the Engineering and Physical Sciences Research Council (Award Reference 1646937) as part of the Doctoral Training program at the University of Liverpool.

$\bowtie \quad$ Aeran Fleming

A.fleming2@liverpool.ac.uk

1 Department of Mathematical Sciences, The University of Liverpool, Mathematical Sciences Building, Liverpool L69 7ZL, UK 
Conjecture 1.2 Let $P_{1}, \ldots, P_{k}$ be points of $\mathbb{P}^{2}$ in general position then for $k \geqslant 9$ the multipoint Seshadri constant

$$
\epsilon\left(\mathbb{P}^{2}, \mathcal{O}_{\mathbb{P}^{2}}(1), P_{1}, \ldots, P_{k}\right)=\frac{1}{\sqrt{k}}
$$

Motivated by work of Biran [2], who proved a symplectic analagon between Nagata's conjecture formulated as a packing problem, Eckl proved in [4] that Nagata's conjecture is in fact equivalent to a more restricted packing problem, namely a Kähler packing problem. More generally in dimension 2 there is a direct correspondence between sizes of multi-ball Kähler packings and multipoint Seshadri constants. A similar result was obtained by Witt Nyström in [9] but this time for a variety of any dimension blown up at a single point. The aim of this note is to generalise these results to projective complex manifolds of arbitrary dimension blown up at any number of points (in general position or not). During the writing of this paper a similar result was achieved by Trusiani in [8] however there are some differences in the formulation of the statement and its proof. In more details:

Definition 1.3 Let $(X, \omega)$ be an $n$-dimensional Kähler manifold with Kähler form $\omega$. Then a holomorphic embedding

$$
\phi=\coprod_{q=1}^{k} \phi_{q}: \coprod_{q=1}^{k} B_{0}\left(r_{q}\right) \rightarrow X
$$

is called a Kähler embedding of $k$ disjoint complex flat balls in $\mathbb{C}^{n}$ centered in 0 , of radius $r_{q}$, if there exists a Kähler form $\omega^{\prime}$ such that $\left[\omega^{\prime}\right]=c_{1}(L)$ and $\phi_{q}^{*}\left(\omega^{\prime}\right)=\omega_{\text {std }}$ is the standard Kähler form on $\mathbb{C}^{n}$ restricted to $B_{0}\left(r_{q}\right)$. Let

$$
\begin{aligned}
& \gamma_{k}\left(X, \omega ; P_{1}, \ldots, P_{n}\right) \\
& \quad=\sup \left\{r>0 \text { : there exists a Kähler packing with } \phi_{q}(0)=P_{q}\right\} .
\end{aligned}
$$

We call $\gamma_{k}$ the $k$-ball packing constant.

Since curvature is an invariant under open holomorphic embeddings we cannot define the packing condition $\phi_{q}^{*}(\omega)=\omega_{\text {std }}$ using the original Kähler form $\omega$ on $X$ as long as that form is not flat enough around $q$. However the following theorem shows that under suitable conditions it is possible to find a Kähler form flat enough around $q$ in the cohomology class of $\omega$ as requested in the definition.

Theorem 1.4 With the notation as above we have that the square of the $k$-ball packing constant is equal to the multipoint Seshadri constant:

$$
\gamma_{k}\left(X, \omega ; P_{1}, \ldots, P_{k}\right)=\sqrt{\epsilon\left(X, L ; P_{1}, \ldots, P_{k}\right)} .
$$




\section{Multipoint Seshadri constants}

In this section we start by recalling some facts and definitions related to Seshadri constants. Single point Seshadri constants have been quite well studied and a good introduction to them can be found in [6, Chapter 5] and [1]. As we are interested in multipoint Seshadri constants we will introduce the notation and some ideas needed later.

Let $X$ be a complex projective manifold of dimension $n$, with $P_{1}, \ldots, P_{k}$ distinct points of $X$. Let $L$ be an ample divisor and $\widetilde{X}:=\mathrm{Bl}_{P_{1}, \ldots, P_{k}}(X) \stackrel{\pi}{\rightarrow} X$ be the blowup of $X$ at the points $P_{1}, \ldots, P_{k}$. Let $\pi^{-1}\left(P_{i}\right)=E_{i}$ denote the exceptional divisor corresponding to the point $P_{i}$ for all $i=1, \ldots, k$ and for $\epsilon_{i} \in \mathbb{Q}_{>0}$ set

$$
\widetilde{L}:=\pi^{*} L-\sum_{i=1}^{k} \epsilon_{i} E_{i} \text { for } \epsilon_{i} \in \mathbb{Q}_{>0} .
$$

If $L$ is a nef $\mathbb{Q}$-Cartier divisor then [6] defines the multipoint Seshadri constant

$$
\epsilon\left(X, L ; P_{1}, \ldots, P_{k}\right)=\max \left\{\epsilon \geqslant 0: \pi^{*} L-\sum_{i=1}^{k} \epsilon_{i} E_{i} \text { is nef }\right\}
$$

Since we require $L$ ample to achieve the desired Kähler packing we show that there is an equivalent definition where we replace max with sup and $\geqslant 0$ with $>0$. The following lemmas prove that the two definitions are equivalent.

Lemma 2.1 Assume that $L$ is ample on $X$. Then $\pi^{*} L-\epsilon^{\prime} E$ is ample on $\tilde{X} \stackrel{\pi}{\rightarrow} X$ if $0<\epsilon^{\prime}<\epsilon(X, L ; P)$.

Proof By Seshadri's Criterion [6, Theorem 1.4.13] if $L$ is ample on $X$ then there exists $\epsilon_{L}>0$ such that for all points $Q \in X$ and all irreducible curves $C$ containg $Q$ we have that $\frac{L \cdot C}{\text { mult }_{Q} C}>\epsilon_{L}$. Let $\widetilde{Q} \in \widetilde{X}$ be such that $\pi(\widetilde{Q})=Q$ and let $\widetilde{C} \subset \widetilde{X}$ be an irreducible curve with $\widetilde{Q} \in \widetilde{C}$. Then there are the following cases. If $\widetilde{Q} \notin E$ then

$$
\frac{\left(\pi^{*} L-\epsilon^{\prime} E\right) \cdot \widetilde{C}}{\operatorname{mult}_{\widetilde{Q}} \widetilde{C}}=\frac{L \cdot C}{\operatorname{mult}_{Q} C}-\epsilon^{\prime} \frac{\text { mult }_{P} C}{\text { mult }_{Q} C} \geqslant \frac{\epsilon_{L}}{2} \quad \text { if } \frac{\text { mult }_{P} C}{\text { mult }_{Q} C}<\frac{\epsilon_{L}}{2 \epsilon^{\prime}}
$$

On the other hand, if $\frac{\text { mult } P_{P} C}{\text { mult }_{Q} C} \geqslant \frac{\epsilon_{L}}{2 \epsilon^{\prime}}$ then

$$
\begin{aligned}
\frac{\left(\pi^{*} L-\epsilon^{\prime} E\right) \cdot \widetilde{C}}{\operatorname{mult}_{\widetilde{Q}} \widetilde{C}} & =\frac{\left(\pi^{*} L-\epsilon_{P} E\right) \cdot \widetilde{C}}{\operatorname{mult}_{\widetilde{Q}} \widetilde{C}}+\left(\epsilon_{P}-\epsilon^{\prime}\right) \frac{E \cdot \widetilde{C}}{\text { mult } \widetilde{Q}^{C}} \\
& \geqslant\left(\epsilon_{P}-\epsilon^{\prime}\right) \frac{\operatorname{mult}_{P} C}{\operatorname{mult}_{Q} C} \geqslant\left(\epsilon_{P}-\epsilon^{\prime}\right) \frac{\epsilon_{L}}{2 \epsilon^{\prime}} .
\end{aligned}
$$


If $\widetilde{Q} \in E$ with $\widetilde{C} \nsubseteq E$ then mult $\widetilde{Q} \widetilde{C} \leqslant$ mult $_{P} \pi(\widetilde{C})$ and

$$
\frac{\left(\pi^{*} L-\epsilon^{\prime} E\right) \cdot \widetilde{C}}{\operatorname{mult}_{\widetilde{Q}} \widetilde{C}} \geqslant \frac{\left(\pi^{*} L-\epsilon^{\prime} E\right) \cdot \widetilde{C}}{\operatorname{mult}_{P} \pi(\widetilde{C})} \geqslant \epsilon_{P}-\epsilon^{\prime}>0 .
$$

Finally if $\widetilde{Q} \in E$ and $\widetilde{C} \subset E$ then

$$
\frac{\left(\pi^{*} L-\epsilon^{\prime} E\right) \cdot \widetilde{C}}{\operatorname{mult}_{\widetilde{Q}} \widetilde{C}} \geqslant-\frac{\epsilon^{\prime} E \cdot \widetilde{C}}{\operatorname{mult}_{\widetilde{Q}} \widetilde{C}} \geqslant \epsilon^{\prime}
$$

The final inequality appears, since $E \cdot \widetilde{C}=\operatorname{deg} \widetilde{C}$ on $E \cong \mathbb{P}^{n-1}$ and $\operatorname{deg} \widetilde{C} \geqslant \operatorname{mult} \widetilde{Q} \widetilde{C}$. Hence we have shown that if $0<\epsilon^{\prime}<\epsilon_{P}$ then $\pi^{*} L-\epsilon^{\prime} E$ is ample.

Corollary $2.2 \max \left\{\epsilon \geqslant 0: \pi^{*} L-\epsilon E\right.$ is nef $\}=\sup \left\{\epsilon>0: \pi^{*} L-\epsilon E\right.$ is $\mathbb{Q}$-ample $\}$.

Lemma 2.3 There exist $\epsilon_{1}, \ldots, \epsilon_{k}>0$ such that $\widetilde{L}$ is $\mathbb{Q}$-ample for all $i=1, \ldots, k$.

Proof By Seshadri's Criterion and Lemma 2.1 we know there exists some $\epsilon_{1}>0$ such that $\pi^{*} L-\epsilon_{1} E$ is $\mathbb{Q}$-ample on $\widetilde{X}_{1}=\mathrm{Bl}_{P_{1}}(X)$. Choosing a second point $P_{2} \in X$ Seshadri's Criterion and Lemma 2.1 again ensure the existence of some $\epsilon_{2}>0$ such that $\pi^{*} L-\epsilon_{1} E_{1}-\epsilon_{2} E_{2}$ is $\mathbb{Q}$-ample on $\widetilde{X}_{2}=\mathrm{Bl}_{P_{1}, P_{2}}(X)$. Arguing iteratively for $k$ points of $X$ we obtain the claim of the lemma.

From the statement of the above lemma it is not obvious that we can chose all the $\epsilon_{i}$ to be equal. This follows from the next lemma.

Lemma 2.4 If $\pi_{k}^{*} L-\sum_{i=1}^{k} \epsilon_{i} E_{i}$ is $\mathbb{Q}$-ample then $\pi^{*} L-\sum_{i=1}^{k} \epsilon_{i}^{\prime} E_{i}$ is ample if $0<$ $\epsilon_{i}^{\prime} \leqslant \epsilon_{i}$, for all $i=1, \ldots, k$.

Proof Lemma 2.1 proves the case when $k=1$. If we show that $\pi^{*} L-\sum_{i=1}^{k} \epsilon_{i} E_{i}$ ample implies $\pi_{k-1}^{*} L-\sum_{i=1}^{k-1} \epsilon_{i} E_{i}$ is ample, where $\pi_{k-1}: \tilde{X}_{k-1} \rightarrow X$ denotes the blow-up of $X$ at the first $k-1$ points, then we can simply apply Lemma 2.1 on the last blow-up. Let $C \subset \widetilde{\widetilde{X}}_{k-1}$ be an irreducible curve on $\widetilde{X}_{k-1}$ and $\bar{C} \subset \widetilde{X}_{k}$ be the strict transform of $C$ on $\widetilde{X}_{k}$. Let $Q$ be a point on $C$. Then

$$
\begin{aligned}
\frac{\left(\pi_{k-1}^{*} L-\sum_{i=1}^{k-1} \epsilon_{i} E_{i}\right) \cdot C}{\operatorname{mult}_{Q} C} & =\frac{\left(\pi_{k}^{*} L-\sum_{i=1}^{k} \epsilon_{i} E_{i}\right) \cdot \widetilde{C}}{\operatorname{mult}_{Q} C}+\frac{\epsilon_{k} E_{k} \cdot \bar{C}}{\text { mult }_{Q} C} \\
& =\frac{\left(\pi_{k}^{*} L-\sum_{i=1}^{k} \epsilon_{i} E_{i}\right) \cdot \widetilde{C}}{\operatorname{mult}_{Q} C}+\epsilon_{k} \cdot \frac{\operatorname{mult}_{P_{k}} C}{\text { mult }_{Q} C}
\end{aligned}
$$

If $Q \neq P_{k}$ we have mult $Q=$ mult $_{Q} \widetilde{C}$, so the sum above is $\geqslant \epsilon_{\pi^{*} L-\sum_{i=1}^{k} \epsilon_{i} E_{i}}$, the global Seshadri constant of $\pi_{k}^{*} L-\sum_{i=1}^{k} \epsilon_{i} E_{i}$. If $Q=P_{k}$, the sum above is $\geqslant \epsilon_{k}$.

Thus, Seshadri's Criterion implies the ampleness of $\pi_{k-1}^{*} L-\sum_{i=1}^{k-1} \epsilon_{i} E_{i}$. 
Definition 2.5 For $X$ and $L$ defined as above the multipoint Seshadri constant associated to points $P_{1}, \ldots P_{k}$ of $\mathrm{X}$ is

$$
\epsilon\left(X, L ; P_{1}, \ldots, P_{k}\right)=\sup \left\{\epsilon \in \mathbb{Q}_{>0}: \pi^{*} L-\epsilon \sum_{i=1}^{k} E_{i} \text { is } \mathbb{Q} \text {-ample }\right\} .
$$

The multipoint Seshadri constant associated to an ample divisor is always $>0$ by Lemma 2.4.

\section{Degeneration of complex projective manifolds to multipoint blow-ups}

First we fix some general notation and introduce some constructions that will be used later. The notation fixed in this section will be used for the remainder of the report unless otherwise stated.

Let $X$ and $L$ be defined as above and consider the product manifold $X$.

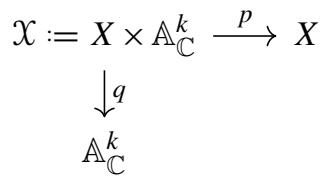

Then if $t_{1}, \ldots, t_{k}$ represent coordinates of $\mathbb{A}_{\mathbb{C}}^{k}$, set $Z_{i}:=\left\{P_{i}\right\} \times\left\{t_{i}=0\right\}$. These are exactly the coordinate hyperplanes cut out by $t_{i}=0$ over the points $P_{i}$.

Remark 3.1 For all $1 \leqslant i<j \leqslant k$ we have $Z_{i} \cap Z_{j}=\varnothing$ since the points $P_{i} \in X$ are distinct.

By blowing up the family $\mathcal{X}$ over the union of all the centres $Z_{i}$ we obtain a new algebraic family over $\mathbb{A}_{\mathbb{C}}^{k}$

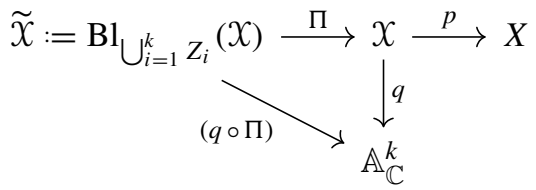

such that the exceptional divisor corresponding to the centre $Z_{i}$ is $\mathcal{E}_{i}:=\Pi^{-1}\left(Z_{i}\right)$. Set $\widetilde{\mathcal{L}}_{d m_{i}, \ldots, m_{k}}=d \Pi^{*} p_{1}^{*} L-\sum_{i=1}^{k} m_{i} \mathcal{E}_{i}$ a divisor on $\tilde{X}$.

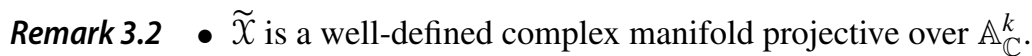

- $\varepsilon_{i} \cong Z_{i} \times \mathbb{P}_{\mathbb{C}}^{n}$.

- $(q \circ \Pi)^{-1}\left(\left(t_{1}, \ldots, t_{k}\right)\right)$ contains the blow-up of $X$ in the points $P_{i}$ where $t_{i}=0$. When $k=1$ the construction is the deformation to the normal cone and the central fibre has two components: the blow-up of $X$ and the exceptional divisor $\mathcal{E}$. 


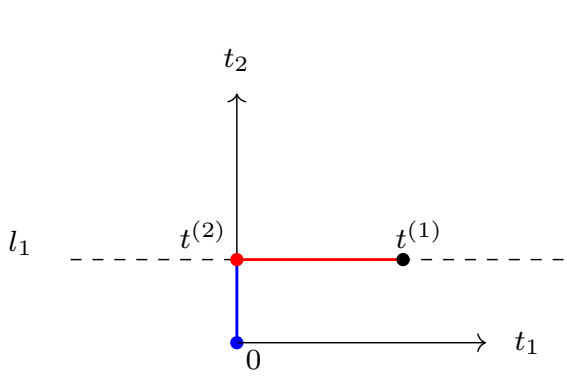

(a) Parameter space for $k=2$

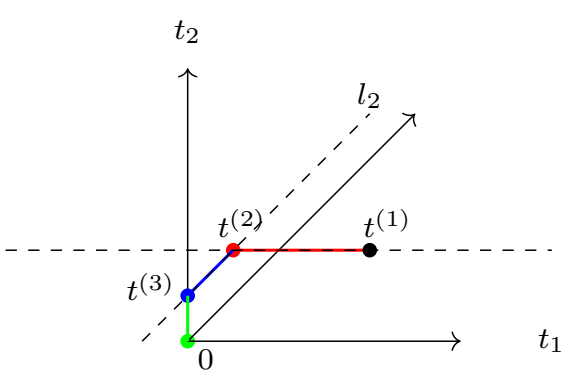

(b) Parameter space for $k=3$

Fig. 1 Illustration of parameter spaces for $k=2$ and $k=3$.

Running through an appropriate path through the parameter space $\mathbb{A}_{\mathbb{C}}^{k}$ these blow-ups accumulate iteratively. Let $\delta_{1}, \ldots, \delta_{k}$ be positive real numbers and define lines in $\mathbb{A}_{\mathbb{C}}^{k}$,

$$
l_{i}:=\left\{\left(0, \ldots, 0, t \cdot \delta_{i}, \delta_{i+1}, \ldots, \delta_{k}\right): t \in \mathbb{R}\right\}
$$

consisting of points whose first $i-1$ coordinates are zero, the $i$ th coordinate is of the form $t \cdot \delta_{i}$ and the remaining $i+1$ coordinates are constant. For the point $t^{(i)}=$ $\left(0, \ldots, 0, \delta_{i}, \delta_{i+1}, \ldots, \delta_{k}\right) \in l_{i}$ for $i=1, \ldots, k$, the preimage is

$$
(q \circ \Pi)^{-1}\left(t^{(i)}\right)=\mathrm{Bl}_{P_{1}, \ldots, P_{i-1}}(X) \cup \bigcup_{j=1}^{i-1} \varepsilon_{j} \cap(q \circ \Pi)^{-1}\left(t^{(i)}\right) .
$$

Following the line from $l_{i}$ from $t^{(i)}$ to $t^{(i+1)}$ we trace a path through the parameter space such that the preimage of $t^{(1)}$ is simply $X$, the preimage of $t^{(2)}$ is simply the blow-up of $X$ at $P_{1}$ with some contributions from the exceptional divisor and so on until we get that the preimage of $t^{(k+1)}=(0, \ldots, 0)$ is the blow-up of $X$ at $P_{1}, \ldots, P_{k}$. See Fig. 1 for a visualisation.

Similarly for $l_{i} \cong \mathbb{A}_{\mathbb{C}}^{1}$ we have

$$
(q \circ \Pi)^{-1}\left(l_{i}\right)=\left(\bigcup_{j=1}^{i-1} \varepsilon_{j} \cap(q \circ \Pi)^{-1}\left(l_{i}\right)\right) \cup \widetilde{X}_{i} .
$$

Where $\widetilde{X}_{i}$ is the blow-up of $\widetilde{X}_{i-1} \times l_{i}$ in the point $\left(P_{i}, 0\right)$.

$$
\begin{aligned}
& \tilde{x}_{i} \stackrel{\prod_{\Pi_{i}} x_{i}=\tilde{X}_{i-1} \times l_{i} \stackrel{q_{i-1}}{\longrightarrow} l_{i} \cong \mathbb{A}_{\mathbb{C}}^{1}}{\Pi^{(i)}} \\
& \downarrow^{p_{i-1}} \\
& \tilde{X}_{i-1}
\end{aligned}
$$


On $\widetilde{X}_{i}$ there exists a family of divisors

$$
\widetilde{\mathcal{L}}_{d, m_{1}, \ldots, m_{i}}^{(i)}:=d \Pi_{i}^{*} p_{i-1}^{*} \widetilde{L}_{d ; m_{1}, \ldots, m_{i-1}}^{i-1}-m_{i} \mathcal{E}_{i}^{(i)}
$$

where $\mathcal{E}_{i}^{(i)} \cong \mathbb{P}_{\mathbb{C}}^{n}$ is the exceptional divisor of the blow-up $\Pi_{i}$. Specific divisors associated to a particular fiber are denoted $\widetilde{\mathcal{L}}_{d, \underline{m}, t}^{(i)}$, where $t$ denotes the parameter on $\mathbb{A}_{\mathbb{C}}^{1}$ and $d, \underline{m}_{i}=\left(m_{1}, \ldots, m_{i}\right)$ record the degree and multiplicity (we will often just write $\tilde{\mathcal{L}}_{t}^{(i)}$ for short if the multiplicity and degree are fixed).

Remark 3.3 $\tilde{X}_{i, 0}=\widetilde{X}_{i} \cup \mathcal{E}_{i}^{(i)}$, where $\mathcal{E}_{i}^{(i)} \cong \mathbb{P}_{\mathbb{C}}^{n}$ and $\tilde{X}_{i, t}=\Pi^{(i)-1}(t)$ is the fiber over $l_{i} \cong \mathbb{A}_{\mathbb{C}}^{1}$

Now that we have defined an algebraic family $\widetilde{X}_{i}$ and a family of divisors $\widetilde{\mathcal{L}}^{(i)}=\widetilde{\mathcal{L}}_{d ; m_{i}}^{(i)}$ we would like to investigate the global sections of these divisors restricted to the fibers of $\Pi^{(i)}$, and how these global sections are constructed for different $i$. This is shown by the following theorem.

Theorem 3.4 For all $i=1, \ldots, k$ there exist global sections $\sigma_{0}^{(i-1)}, \ldots, \sigma_{N_{i}}^{(i-1)}$ of $\Pi_{*}^{(i)} \widetilde{\mathcal{L}}^{(i)}$ such that:

(1) $\sigma_{0}^{(i-1)}, \ldots, \sigma_{N_{i}}^{(i-1)}$ trivialise $\Pi_{*}^{(i)} \widetilde{\mathcal{L}}^{(i)}$.

(2) If $0_{i}$ denotes the zero of the line $l_{i}$ then the sections

$$
\left.\sigma_{0,0_{i}}^{(i-1)}\right|_{\mathcal{E}_{i}^{(i)}}, \quad \ldots,\left.\quad \sigma_{N_{i}, 0_{i}}^{(i-1)}\right|_{\mathcal{E}_{i}^{(i)}}
$$

generate the Kähler form $m_{i} \cdot \omega_{\mathrm{FS}}$ on $\mathcal{E}_{i}^{(i)} \cong \mathbb{P}_{\mathbb{C}^{-}}^{n}$

(3) If $i \geqslant 2$ then the restricted sections $\sigma_{0, \delta_{i}}^{(i-1)}, \ldots, \sigma_{N_{i}, \delta_{i}}^{(i-1)}$ generate the same Kähler form on $\widetilde{X}_{i, \delta_{i}} \cong \widetilde{X}_{i-1}$ as $\left.\sigma_{0,0_{i-1}}^{(i-2)}\right|_{\tilde{X}_{i-1}}, \ldots, \sigma_{N_{i-2}, 0_{i-1}}^{(i-2)} \mid \tilde{X}_{i-1}$ from the previous family $\tilde{x}_{i-1}$.

Proof First we illustrate the special case when $i=k=1$. Let $X$ be a complex projective manifold equipped with an ample line bundle $L$ and fix the point $P_{1}$. Let $\pi: \widetilde{X}_{1}=\mathrm{Bl}_{P_{1}}(X) \rightarrow X$ be the blow-up of $X$ at $P_{1}$, with $E_{1}=\pi^{-1}\left(P_{1}\right)$ the exceptional divisor and choose positive integers $m$ and $d$ such that $\widetilde{L}^{(1)}=d \pi^{*} L-m E$ is ample (in particular, $m<d$ ). Now construct the families $X$ and $\widetilde{X}_{1}$ using the strategy detailed above (see Fig. 2).

Recall that on $\widetilde{X}_{1}$ there exists a family of ample line bundles $\widetilde{L}^{(1)}$ and the central fiber $\widetilde{X}_{0} \cong \widetilde{X}_{1} \cup \mathcal{E}_{1}$ where $\varepsilon_{1} \cong \mathbb{P}^{n}$. In this setting we can determine a basis of sections of $H^{0}\left(\widetilde{X}_{0}, \widetilde{L}^{(1)} \mid \widetilde{X}_{0}\right)$ characterised by vanishing behaviour at the point $P_{1}$ and show that they satisfy the requirements of the theorem. First we compute sections of $H^{0}\left(\widetilde{X}_{1}, \mathcal{O}_{\widetilde{X}}\left(\widetilde{L}^{(1)}\right)\right)$ vanishing to order greater or equal $m$ in $P_{1}$. These sections are homogeneous polynomials of degree less than or equal $m$ described in local coordinates around $P_{1}$. Sections of $H^{0}\left(\mathcal{E}_{1}, \mathcal{O}_{\mathcal{E}_{1}}\left(-m E_{1}\right)\right)$ are simply homogeneous monomials 


$$
\widetilde{x}_{1}=\tilde{X}_{1} \times \mathbb{A}_{\mathbb{C}}^{1}
$$

$x=X \times \mathbb{A}_{\mathbb{C}}^{1}$
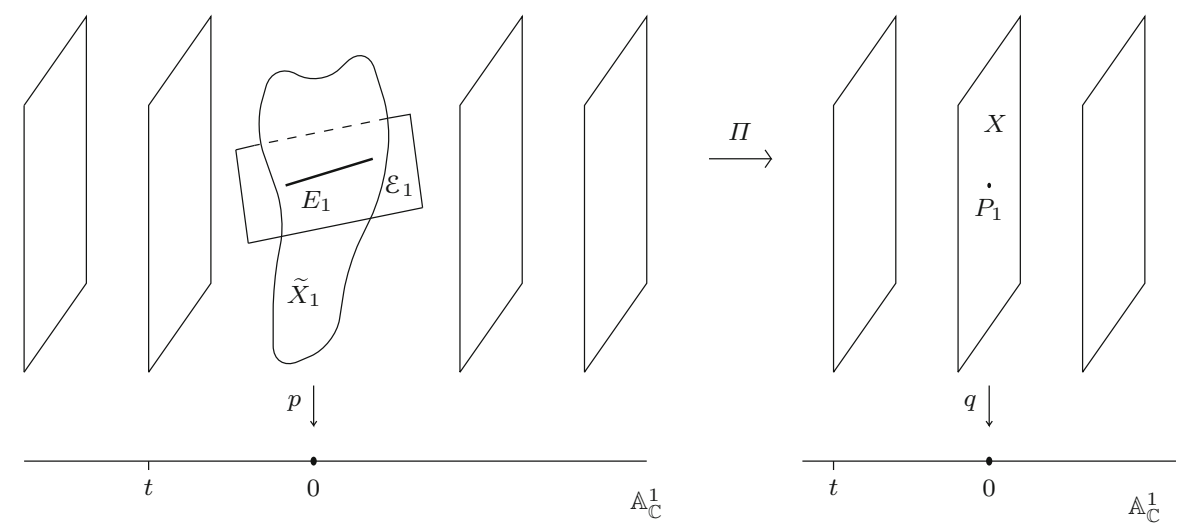

Fig. 2 Product manifold and blow-up for $i=k=1$.

of degree $m$ on $\mathbb{P}^{n}$ under the isomorphism $\varepsilon_{1} \cong \mathbb{P}^{n}$. These monomials restrict to monomials on $E_{1}$ if they vanish with multiplicity greater than $m$ and to 0 otherwise. Similarly the section of $\widetilde{L}^{(1)}$ restricts to a homogeneous monomial on $E_{1}$ if it vanishes with multiplicity greater than $m$ and 0 otherwise. These sections form a basis of $H^{0}\left(\widetilde{X}_{1}, \mathcal{O}_{\widetilde{X}_{1}}\left(\widetilde{L}^{(1)}\right)\right)$ and to change between a basis of sections on $\widetilde{X}_{1}$ and on $X$ we simply skip those sections which vanish to order less than $m$ at $P_{1}$.

To obtain sections of $\mathcal{L}^{(1)}$ we pull back a section $s \in H^{0}\left(\widetilde{X}_{1}, \mathcal{O}_{\widetilde{X}_{1}}\left(\widetilde{L}^{(1)}\right)\right)$ along $p$ to get a section of $p^{*} \widetilde{L}^{(1)}=\mathcal{L}^{(1)}$ on $\widetilde{X}_{1}=\widetilde{X}_{1} \times \mathbb{A}_{\mathbb{C}}^{1}$. If mult $P_{1} s<m$ then the section of $H^{0}\left(\widetilde{X}_{1}, \widetilde{\mathcal{L}}^{(1)}\right)$ has multiplicity $m$ in $\left(P_{1}, 0\right)$ and if mult $P_{1} s \geqslant m$ then $\operatorname{mult}_{\left(P_{1}, 0\right)} p^{*} s \geqslant$ $m$. In both cases, we can subtract $m$ copies of the exceptional divisor $\mathcal{E}_{1}$ from the pullback of this section along $\Pi$, thus obtaining sections of $H^{0}\left(\widetilde{X}_{1}, \widetilde{L}^{(1)}\right)$.

By construction the general fiber $\widetilde{X}_{1, t}$ is isomorphic to $X$ for $t \neq 0$, so it is clear that away from the central fiber the sections described above trivialise $\Pi_{*} \widetilde{\mathcal{L}}^{(1)}$. Considering the central fiber we find that global sections of $\widetilde{\mathcal{L}}^{(1)} \mid \tilde{X}_{1,0}$ consist of a section of $\widetilde{\mathcal{L}}^{(1)} \mid \widetilde{X}_{1}$ and a section of $\left.\tilde{\mathcal{L}}^{(1)}\right|_{\mathcal{E}_{1}}$ coinciding when restricted to $E_{1}$. These sections are comprise of:

- pairs of basis sections on $\widetilde{X}_{1}$ vanishing on $E_{1}$ and the zero section on $\varepsilon_{1}$,

- pairs of the zero section on $\widetilde{X}_{1}$ and a basis section on $\mathcal{E}_{1}$ vanishing on $E_{1}$,

- pairs of basis sections of $\widetilde{X}_{1}$ and $\varepsilon_{1}$ that restrict to the same non-zero homogeneous polynomial of degree $m$ on $E_{1}$.

These sections all coincide to basis sections of $d L$ which either: vanish to multiplicity greater than $m$ at $P_{1}$, vanish to exactly order $m$ at $P_{1}$, or vanish to order less than $m$ at $P_{1}$. Hence these sections restrict to a basis of $\widetilde{X}_{1,0}$ and therefore trivialise $\Pi_{*} \widetilde{\mathcal{L}}^{(1)}$ over all fibers of the family.

To prove the general case we show that the dimension of the space of global sections of $\widetilde{\mathcal{L}}^{(i)}$ restricted to each fiber of $\Pi^{(i)}$ is the same, Grauert's semi-continuity theorem 
[5, III.12] implies immediately that $\Pi_{*}^{(i)} \widetilde{\mathcal{L}}^{(i)}$ is locally free, hence it is free by the Quillen-Suslin Theorem. Thus there exist trivialising global sections of $\Pi^{*} \widetilde{\mathcal{L}}^{(i)}$. But instead of using these powerful theorems we describe the trivialising sections directly because they allow us to prove the properties of the theorem more easily. For the sections to be trivialising it is enough to show that they form a basis of $H^{0}\left(\widetilde{\mathcal{X}}_{i, t}, \widetilde{\mathcal{L}}_{t}^{(i)}\right)$ then restricted to $\widetilde{X}_{i, t}$ for all $t \in \mathbb{A}_{\mathbb{C}}^{1}$.

We start by calculating a basis of sections of $H^{0}\left(X, \mathcal{O}_{X}(d L)\right)$ characterised by their vanishing behaviour at the points $P_{1}, \ldots, P_{k}$ so that subsets of this basis can be interpreted as generating sections of $H^{0}\left(\widetilde{X}, \widetilde{L}^{(i)}\right)$. Let $\mathfrak{m}_{X, P_{i}}$ denote the maximal ideal of $X$ at $P_{i}$ and consider the short exact sequence

$$
0 \rightarrow \bigcap_{i=1}^{k} \mathfrak{m}_{X, P_{i}}^{m_{i}+1} \otimes \mathcal{O}_{X}(d L) \rightarrow \mathcal{O}_{X}(d L) \rightarrow \bigoplus_{i=1}^{k} \mathcal{O}_{X} / \mathfrak{m}_{X_{i}, P_{i}}^{m_{i}+1} \rightarrow 0
$$

Taking the long exact sequence of cohomology w.r.t. to the above sequence gives

$$
\begin{aligned}
& 0 \longrightarrow H^{0}\left(X, \bigcap_{i=1}^{k} \mathfrak{m}_{X, P_{i}}^{m_{i}+1} \otimes \mathcal{O}_{X}(d L)\right) \rightarrow H^{0}\left(X, O_{X}(d L)\right) \longrightarrow H^{0}\left(X, \bigoplus_{i=1}^{k} \mathcal{O}_{X} / \mathfrak{m}_{X, P_{i}}^{m_{i}+1}\right) \\
& \longrightarrow H^{1}\left(X, \bigcap_{i=1}^{k} \mathfrak{m}_{X, P_{i}}^{m_{i}+1} \otimes \mathcal{O}_{X}(d L)\right) \longrightarrow H^{1}\left(X, \mathcal{O}_{X}(d L)\right) \longrightarrow \ldots \ldots \ldots \ldots
\end{aligned}
$$

By Serre Vanishing $H^{1}\left(X, \bigcap_{i=1}^{k} \mathfrak{m}_{X, P_{i}}^{m_{i}+1} \otimes \mathcal{O}_{X}(d L)\right)=0$, since applying the projection formula yields

$$
H^{1}\left(X, \bigcap_{i=1}^{k} \mathfrak{m}_{X, P_{i}}^{m_{i}+1} \otimes \mathcal{O}_{X}(d L)\right)=H^{1}\left(\tilde{X}, \mathcal{O}_{X}\left(\widetilde{L}_{d, m_{i}}\right)\right)
$$

and we assume $\widetilde{L}_{d, m_{i}}$ is ample and $d, \underline{m}_{k} \gg 0$. Hence $\phi: H^{0}\left(X, \mathcal{O}_{X}(d L)\right) \rightarrow$ $H^{0}\left(X, \bigoplus_{i=1}^{k} \mathcal{O}_{X} / \mathfrak{m}_{X, P_{i}}^{m_{i}+1}\right)$ is surjective. This gives a basis of sections of $H^{0}\left(X, \mathcal{O}_{X}(d L)\right)$ which is the union of:

- A basis $B_{0}$ of $H^{0}\left(X, \bigcap_{i=1}^{k} \mathfrak{m}_{X, P_{i}}^{m_{i}+1} \otimes \mathcal{O}_{X}(d L)\right)$, which are sections of $\mathcal{O}_{X}(d L)$ vanishing to multiplicity at least $m_{i}+1$ in $P_{i}$ for $i=1, \ldots, k$.

- A set $B_{i}$ of sections of $\mathcal{O}_{X}(d L)$ mapped to 0 in $\mathcal{O}_{X} / \mathfrak{m}_{X, P_{j}}^{m_{j}^{+1}}$ for $j \neq i$ and to a basis of homogeneous polynomials of degree $\leqslant m_{i}$ in $\mathcal{O}_{X} / \mathfrak{m}_{X, P_{i}}^{m_{i}+1}$, in variables given by local coordinates around $P_{i}$.

- A set of sections $\widetilde{B}_{i}$ of $\mathcal{O}_{X}(d L)$ as above, but with homogeneous polynomials of degree $=m_{i}$, and that $\widetilde{B}_{i} \subset B_{i}$.

Hence a basis of $H^{0}\left(\widetilde{X}_{i}, \mathcal{O}_{\tilde{X}_{i}}\left(\widetilde{L}^{(i)}\right)\right)$ is given by

$$
B^{(i)}:=B_{0} \cup \bigcup_{j=1}^{i} \widetilde{B}_{j} \cup \bigcup_{j=i+1}^{k} B_{j}
$$


To change between a basis of sections on $\widetilde{X}_{i-1}$ and on $\widetilde{X}_{i}$ we simply skip those sections which vanish to order less than $m_{i}$ at $P_{i}$.

Since $X_{i}=\widetilde{X}_{i-1} \times \mathbb{A}_{\mathbb{C}}^{1}$, we can use these sections to construct the sections of $H^{0}\left(\widetilde{X}_{i}, \widetilde{\mathcal{L}}^{(i)}\right)$ using the following procedure:

Step 1: Pull back a section $s \in H^{0}\left(\widetilde{X}_{i-1}, \mathcal{O}_{X_{i-1}}\left(\widetilde{L}^{(i-1)}\right)\right)$ along $p_{i-1}$ to get a section of $p_{i-1}^{*} \widetilde{L}^{(i-1)}=\mathcal{L}^{(i-1)}$ on $X_{i}=\widetilde{X}_{i-1} \times \mathbb{A}_{\mathbb{C}}^{1}$.

Step 2: If mult $P_{i} s<m_{i}$ then the section $t^{m_{i}-\operatorname{mult}_{P_{i}} s} p_{i-1}^{*} s \in H^{0}\left(\widetilde{\mathcal{X}}_{i}, \widetilde{\mathcal{L}}^{(i-1)}\right)$ has multiplicity $m_{i}$ in $\left(P_{i}, 0\right)$. If mult $P_{i} s \geqslant m_{i}$ then $\operatorname{mult}_{\left(P_{i}, 0\right)} p_{i-1}^{*} s \geqslant m_{i}$.

Step 3: In both cases, we can subtract $m_{i}$ copies of the exceptional divisor $\mathcal{E}_{i}$ from the pullback of this section along $\Pi_{i}$, thus obtaining sections of $H^{0}\left(\widetilde{\mathcal{X}}_{i}, \widetilde{\mathcal{L}}^{(i)}\right)$.

When starting with the basis sections in $B^{(i-1)}$, restricting to the general fibers $\widetilde{X}_{i, t} \cong$ $\widetilde{X}_{i-1}$ of $\Pi^{(i)}$ (that is $t \neq 0$ ) we get back the sections in $B^{(i-1)}$ possibly multiplied with some power of $t$. Thus all the sections $\sigma_{1}^{(i-1)}, \ldots \sigma_{N_{i}}^{(i-1)}$ obtained from $B^{(i-1)}$ in the way described above are trivializing $\Pi_{*}^{(i)} \widetilde{\mathcal{L}}^{(i)}$ outside the central fiber. To understand the restriction of these sections to the central fiber we need to analyse $\widetilde{\mathcal{L}}^{(i)} \mid \widetilde{x}_{i, 0}$ and its global sections. Note, $\widetilde{X}_{i, 0}=\widetilde{X}_{i} \cup \mathcal{E}_{i}^{(i)}$ and $\widetilde{X}_{i} \cap \mathcal{E}_{i}^{(i)}=E_{i}$. Furthermore $\widetilde{\mathcal{L}}^{(i)} \mid \widetilde{X}_{i}=\widetilde{L}^{(i)}$, and $\left.\widetilde{\mathcal{L}}^{(i)}\right|_{\mathcal{E}_{i}^{(i)}}=\mathcal{O}_{\mathcal{E}_{i}^{(i)}}\left(-m_{i} \mathcal{E}_{i}^{(i)}\right) \cong \mathcal{O}_{\mathbb{P}^{n}}\left(m_{i}\right)$ via the isomorphism $\mathcal{E}_{i}^{(i)} \cong \mathbb{P}_{\mathbb{C}}^{n}$. This means that global sections of $\widetilde{\mathcal{L}}^{(i)} \mid \widetilde{x}_{i, 0}$ consist of a section of $\left.\widetilde{\mathcal{L}}^{(i)}\right|_{\tilde{X}_{i}}$ and a section of $\left.\widetilde{\mathcal{L}}^{(i)}\right|_{\mathcal{E}_{i}^{(i)}}$ coinciding when restricted to $E_{i}$.

- Global sections of $\widetilde{\mathcal{L}}^{(i)} \mid \tilde{X}_{i}$ and their restriction to $E_{i}$ :

These are all sections of $d L$ on $X$ which vanish to multiplicity $\geqslant m_{j}$ in $P_{j}$ for $j=1, \ldots, i$. Equivalently these are global sections of $\widetilde{L}^{(i-1)}$ on $\widetilde{X}_{i-1}$ vanishing in multiplicity $\geqslant m_{i}$ in $P_{i}$. To obtain a section of $\widetilde{L}^{(i)}$ from a section of $\widetilde{L}^{(i-1)}$ we pull back the section along the map $\pi^{(i-1)}: \widetilde{X}_{i} \rightarrow \widetilde{X}_{i-1}$ then subtract $m_{i}$ copies of the exceptional divisor. If a section of $\left.\widetilde{\mathcal{L}}^{(i)}\right|_{\widetilde{X}_{i}}=\widetilde{L}^{(i)}$ corresponds to a section of $\widetilde{L}^{(i-1)}$ with multiplicity greater than $m_{i}$ at $P_{i}$ the restriction of this section to $E_{i}$ is zero. If the multiplicity is exactly $m_{i}$ at $P_{i}$ then $\left.\widetilde{\mathcal{L}}^{(i)}\right|_{E_{i}} \cong \mathcal{O}_{\mathbb{P}^{n-1}}\left(m_{i}\right)$ hence the restriction of such a section of $\widetilde{\mathcal{L}}^{(i)}$ to $E_{i} \cong \mathbb{P}_{\mathbb{C}}^{n}$ will be described by a non-zero homogeneous polynomials of degree $m_{i}$ in $Y_{1}, \ldots, Y_{n}$.

- Global sections of $\left.\widetilde{\mathcal{L}}^{(i)}\right|_{\mathcal{E}_{i}^{(i)}}$ and their restriction to $E_{i}$ :

To describe these we introduce homogeneous coordinates $\left[T: Y_{1}: \ldots: Y_{n}\right]$ on $\mathcal{E}_{i}^{(i)}$. The coordinates $Y_{1}, \ldots, Y_{n}$ come from local coordinates $y_{1}, \ldots, y_{n}$ around $P_{i}$, the $T$ coordinate comes from the affine base parameter $t$, and $T=0$ describes $E_{i} \subset \mathcal{E}_{i}^{(i)}$. Hence the restriction of a section of $\left.\widetilde{\mathcal{L}}^{(i)}\right|_{\mathcal{E}_{i}^{(i)}}$ to $E_{i}$ is obtained by setting $T=0$. Since $\left.\widetilde{\mathcal{L}}^{(i)}\right|_{\mathcal{E}_{i}^{(i)}} \cong \mathcal{O}_{\mathbb{P}^{n}}\left(m_{i}\right)$, sections of $\left.\widetilde{\mathcal{L}}^{(i)}\right|_{\mathcal{E}_{i}^{(i)}}$ are non-zero homogeneous polynomials of degree $m_{i}$ in $T, Y_{1}, \ldots, Y_{n}$.

Sections of $\widetilde{\mathcal{L}}^{(i)}$ are therefore described by pairs of sections of $\widetilde{X}_{i}$ and $\mathcal{E}_{i}^{(i)}$ both vanishing on $E_{i}$ and pairs of sections of $\tilde{X}_{i}$ and $\mathcal{E}_{i}^{(i)}$ restricting to the same non-zero 
homogeneous polynomial in $Y_{i}, \ldots, Y_{n}$ of degree $m_{i}$ on $E_{i}$. A basis of global sections of $\widetilde{\mathcal{L}}^{(i)} \mid \tilde{x}_{i, 0}$ can therefore be built from the basis of $H^{0}\left(\widetilde{X}_{i}, \widetilde{L}^{(i)}\right)$ and $H^{0}\left(\widetilde{\mathcal{E}}_{i}^{(i)},\left.\widetilde{\mathcal{L}}^{(i)}\right|_{\mathcal{E}_{i}^{(i)}}\right)$ as the set of

- Pairs of basis sections on $\widetilde{X}_{i}$ vanishing on $E_{i}$ and the zero section on $\mathcal{E}_{i}^{(i)}$.

- Pairs of the zero section on $\widetilde{X}_{i}$ and a basis section on $\mathcal{E}_{i}^{(i)}$ vanishing on $E_{i}$.

- Pairs of basis sections of $\tilde{X}_{i}$ and $\mathcal{E}_{i}^{(i)}$ that restrict to the same non-zero homogeneous polynomial of degree $m_{i}$ on $E_{i}$.

Now we want to show that the restriction of the $\sigma_{0}^{(i-1)} \ldots \sigma_{N_{i}}^{(i-1)}$ to $\tilde{X}_{i, 0}$ yields a basis of $\widetilde{\mathcal{L}}^{(i)} \mid \widetilde{X}_{i, 0}$. To this purpose, we follow Steps 1-3 to construct $\sigma_{j}^{(i)}$ from a section of $\widetilde{L}^{(i-1)}$ and then identify the pair of sections describing the restriction to $\widetilde{X}_{i, 0}$. If a section of $\widetilde{L}^{(i-1)}$ vanishes to multiplicity strictly greater than $m_{i}$ at $P_{i}$ then it corresponds to a pair of sections on $\widetilde{\mathcal{L}}^{(i)} \mid \widetilde{X}_{i, 0}$ consisting of a section of $\widetilde{\mathcal{L}}^{(i)}$ that restricts to zero on $E_{i}$, and the zero section on $\mathcal{E}_{i}^{(i)}$. If the section of $\widetilde{L}^{(i-1)}$ on $\widetilde{X}_{i-1}$ vanishes to exactly multiplicity $m_{i}$ at $P_{i}$ then pulling back and subtracting $m_{i}$ copies of the exceptional divisor we obtain non-zero sections on $\widetilde{X}_{i}$ and on $\mathcal{E}_{i}^{(i)}$. Such a section of $\widetilde{L}^{(i-1)}$ corresponds to a pair of sections on $\tilde{X}_{i, 0}$ that restrict to the same non-zero homogeneous monomial on $E_{i}$. Finally if a section $s$ of $\widetilde{L}^{(i-1)}$ on $\widetilde{X}_{i-1}$ vanishes to multiplicity strictly less than $m_{i}$ at $P_{i}$ then after pulling back along $p_{i-1}$ we must multiply by $t^{m_{i}-\operatorname{mult}_{P_{i}} s}$ before we can subtract copies of the exceptional divisor. This type of section corresponds to a pair of sections on $\widetilde{X}_{i, 0}$ consisting of the zero section on $\widetilde{X}_{i}$ and a section on $\mathcal{E}_{i}^{(i)}$ that restricts to zero on $E_{i}$.

In terms of the sets $B_{0}, B_{j}$ and $\widetilde{B}_{j}$, making up the basis $B^{(i)}$ of $\widetilde{L}^{(i)}$ we find that a basis of global sections of $\widetilde{L}^{(i-1)}$ vanishing to multiplicity $>m_{i}$ at $P_{i}$ can be written as $B_{0} \cup \bigcup_{j=1}^{i-1} \widetilde{B}_{j} \cup \bigcup_{i+1}^{k} B_{j}$. A basis of global sections vanishing to multiplicity exactly $m_{i}$ at $P_{i}$ is $\widetilde{B}_{i}$ and finally a basis of global sections vanishing to multiplicity less than $m_{i}$ at $P_{i}$ is given by $B_{i}-\widetilde{B}_{i}$. The union of all three bases provides a basis $B^{(i-1)}$ for $\widetilde{X}_{i, t} \cong \widetilde{X}_{i-1}$. The union of the basis corresponding to all sections of $\widetilde{L}^{(i-1)}$ vanishing to multiplicity less than or equal to $m_{i}$ at $P_{i}$ is isomorphic to a basis of global sections of $\left.\widetilde{\mathcal{L}}^{(i)}\right|_{\mathcal{E}_{i}^{(i)}}$ where the identification is given by the correspondence between homogeneous polynomials in local coordinates around $P_{i}$ and homogeneous coordinates of $\varepsilon_{i}^{(i)} \cong \mathbb{P}_{\mathbb{C}}^{n}$.

Thus, the sections $\sigma_{0}^{(i-1)}, \ldots, \sigma_{N_{i}}^{(i-1)}$ also restrict to a basis of $\widetilde{\mathcal{L}}^{(i)} \mid \widetilde{X}_{i, 0}$, hence trivialize $\Pi_{*}^{(i)} \widetilde{\mathcal{L}}^{(i)}$ over all fibers of the family.

Now let us construct a Kähler form using the trivializing sections of $\widetilde{\mathcal{L}}^{(i)}$ restricted to $\mathcal{E}_{i}^{(i)} \cong \mathbb{P}_{\mathbb{C}}^{n}$. On this exceptional divisor we can choose homogeneous coordinates 
$T, Y_{1}, \ldots Y_{n}$ as above. Then

$$
\begin{aligned}
m_{i} \cdot \omega_{\mathrm{FS}} & =\frac{i}{2 \pi} \partial \bar{\partial} m_{i} \cdot \log \left(Y_{1}{\overline{Y_{1}}}+\cdots+Y_{n} \bar{Y}_{n}+T \bar{T}\right) \\
& =\frac{i}{2 \pi} \partial \bar{\partial} \log \left(Y_{1} \bar{Y}_{1}+\cdots+Y_{n} \bar{Y}_{n}+T \bar{T}\right)^{m_{i}} \\
& =\frac{i}{2 \pi} \partial \bar{\partial} \log \left(Y_{1}^{m_{i}}{\overline{Y_{1}}}^{m_{i}}+m_{i} Y_{1}^{m_{i}-1} \bar{Y}_{1}^{m_{i}-1} Y_{2} \bar{Y}_{2} \ldots\right) \\
& =m_{i} \cdot \omega_{\mathrm{FS}}=\frac{i}{2 \pi} \partial \bar{\partial} \log \left(\sum_{\alpha+\beta=m_{i}} c_{\alpha, \beta} Y^{\alpha} \bar{Y}^{\alpha} T^{\beta} \bar{T}^{\beta}\right),
\end{aligned}
$$

where $c_{a, b}$ are positive integers.

If we choose the sections $\sigma_{j}^{(i-1)}$ that do not vanish on the exceptional divisor (i.e., coming from sections of $\widetilde{L}^{(i-1)}$ vanishing with multiplicity $\leqslant m_{i}$ in $P_{i}$ ) such that they restrict to the basis of monomials $\sqrt{c_{\alpha, \beta}} Y^{\alpha} T^{\beta}$ of the homogeneous polynomials of degree $m_{i}$, then $m_{i} \cdot \omega_{\mathrm{FS}}$ is the Kähler form on $\mathcal{E}_{i}^{(i)}$ generated by the restriction of these sections $\sigma_{j}^{(i-1)}$. Note that $c_{\alpha, \beta}$ is a positive integer, so $\sqrt{c_{\alpha, \beta}}$ is just the usual real square root.

To achieve property (3) we have to construct the trivializing sections of $\widetilde{\mathcal{L}}^{(i)}$ on $\widetilde{X}_{i}$ iteratively, starting with $i=k$. On $\widetilde{X}_{k}$ we construct sections $\sigma_{0}^{(k-1)}, \ldots, \sigma_{N_{k}}^{(k-1)}$ as above, which restricted to $\widetilde{X}_{k, \delta_{k}}$ provides a a basis of sections of $\widetilde{L}^{(k-1)}$ on $\widetilde{X}_{k-1}=$ $\tilde{X}_{k, \delta_{k}}$. We can interpret these sections as sections of $\widetilde{L}^{(k-2)}$ on $\widetilde{X}_{k-2}$ vanishing with multiplicity $\geqslant m_{k-1}$ in $P_{k-1}$, and we can complete these sections to a basis of all sections of $\widetilde{L}^{(k-2)}$ by adding sections which vanish with multiplicity $<m_{k-1}$ in $P_{k-1}$. This basis can be used to construct trivializing sections of $\widetilde{\mathcal{L}}^{(k-1)}$ on $\widetilde{X}_{k-1}$ because by its construction it can be split up into the subsets $B_{0}, B_{k-1}, \widetilde{B}_{k-1}$ and $B^{(k-1)}$. The Kähler form on $\varepsilon_{k-1}^{(k-1)}$ is also as requested since we can choose the completing basis sections of $\widetilde{L}^{(k-2)}$ as required above. Iterating this process for $\widetilde{X}_{k-2}, \ldots, \widetilde{X}_{1}$ we deduce property (3) for each $i=1 \ldots, k$.

\section{Symplectic blow-up of Kähler manifolds}

Before we prove the main result of this paper, for the convenience of the reader we present the construction of symplectic blow-ups in the Kähler setting in more details. It immediately yields one half of Theorem 5.1, by relating Kähler packings to the ampleness of line bundles on the blown-up varieties. We follow McDuff's and Polterovich's original construction in [7], especially emphasizing how to keep track of the Kähler condition.

Consider the triples $\left(\mathbb{C}^{n}, J_{0}, \omega_{\text {std }}\right)$ and $\left(\mathbb{C P}^{n-1}, J_{0}, \omega_{\mathrm{FS}}\right)$, where $\omega_{\text {std }}$ is the standard Euclidean form on $\mathbb{C}^{n}, J_{0}$ is the standard complex structure on both $\mathbb{C}^{n}$ and $\mathbb{C P}^{n-1}$, and $\omega_{\mathrm{FS}}$ is the Fubini-Study form on $\mathbb{C P}^{n-1}$. Let $l \subset \mathbb{C P}^{n-1}$ be a line and fix a point 
$z \in \mathbb{C}^{n}$. Then the complex manifold $\mathcal{M}$

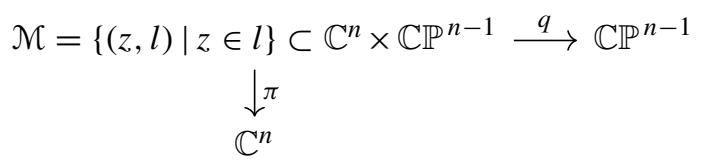

is the blow-up of $\mathbb{C}^{n}$ at the origin. To help the reader visualise this one can imagine replacing the origin of $\mathbb{C}^{n}$ by the set of all complex lines passing through the origin.

Let $B_{\lambda}^{2 n}(0)=\left\{\left(z_{1}, \ldots, z_{n}\right) \in \mathbb{C}^{n}:\left|z_{1}\right|^{2}+\cdots+\left|z_{n}\right|^{2} \leqslant \lambda^{2}\right\}$ denote an $n$ dimensional complex ball of radius $\lambda$ and centred at the origin. For $\lambda>0$ the set $\mathcal{M}(\lambda):=\pi^{-1}\left(B_{\lambda}^{2 n}(0)\right)$ is the sub-manifold obtained by taking the inverse image of $B_{\lambda}^{2 n}(0)$ under the projection $\pi$. We call the fiber $\pi^{-1}(0)$ over 0 the exceptional divisor and the projection $\pi$ the blow-up map. It is worth noting that away from the exceptional divisor $\pi$ is biholomorphic onto $\mathbb{C}^{n}$.

Proposition 4.1 On $\mathcal{M}$ there exists a Kähler form

$$
\rho(1, \lambda):=\pi^{*} \omega_{\text {std }}+\lambda^{2} q^{*} \omega_{\mathrm{FS}} \text { for } \lambda>0 \text {. }
$$

Proof To prove that the bilinear form $\rho(1, \lambda)$ is a Kähler form we must show that it is closed, skew symmetric, that it is compatible with the complex structure on $\mathcal{M}$ and the associated Riemannian metric is positive definite. First we note $\rho(1, \lambda)$ is closed and skew symmetric, as pullbacks of closed and skew symmetric forms are closed and skew symmetric. Since $\omega_{\text {std }}$ and $\omega_{\mathrm{FS}}$ are compatible with the complex structure on $\mathbb{C}^{n}$ and $\mathbb{C P}^{n-1}$ and the projections $\pi$ and $q$ are holomorphic, we can deduce that the associated bilinear form is compatible with the complex structure on $\mathcal{M}$. Finally the associated Riemannian metric is positive definite as the pullbacks are positivesemi definite and furthermore $\pi^{*} \omega_{\text {std }}$ is positive definite away from the exceptional divisor, and positive definite on the tangent vectors mapped to 0 by the differential of $q$. Moreover on the other tangent vectors of $\mathcal{M}, q^{*} \omega_{\mathrm{FS}}$ is positive definite.

Corollary 4.2 On the exceptional divisor $\pi^{-1}(0)$ the $(1,1)$-form $\left.\rho(1, \lambda)\right|_{\pi^{-1}(0)}=$ $\lambda^{2} q^{*} \omega_{\mathrm{FS}}$.

Definition 4.3 An embedding $F: \mathbb{C}^{n}-\{0\} \rightarrow \mathbb{C}^{n}$ is called monotone if in spherical coordinates $(u, r) \in S^{2 n-1} \times(0, \infty) \cong \mathbb{C}^{n}-\{0\}$ it can be written as $(u, r) \mapsto$ $(u, f(r))$, where $f$ is a strictly increasing function.

Lemma 4.4 There exists a smooth family of monotone embeddings

$$
h_{\lambda}: \mathbb{C}^{n}-\{0\} \rightarrow \mathbb{C}^{n}-B(\lambda)
$$

such that $\pi^{*} h_{\lambda}^{*} \omega_{\text {std }}=\rho(1, \lambda)$ on $\mathcal{M}-\pi^{-1}(0)$.

Proof Using spherical coordinates we can choose $h_{\lambda}:(u, v) \mapsto\left(u,\left(r^{2}+\lambda^{2}\right)^{1 / 2}\right)$. Fix a point $P=((c, 0, \ldots, 0),[1: 0: \ldots: 0]) \in \mathcal{M}$ for $c \in \mathbb{R}_{>0}$. Recall that around 
the point $(c, 0, \ldots, 0)$ if we take local coordinates $z_{j}=x_{j}+i y_{j}$ of $\mathbb{C}^{n}$ then $\omega_{\text {std }}=$ $\sum_{j=1}^{n} d x_{j} \wedge d y_{j}$. Furthermore if $t_{j}=u_{j}+i v_{j}$ are local coordinates of $\mathbb{C P}^{n-1}$ around $[1: 0: \ldots: 0]$ then the Fubini-Study form in $P$ is given as $\omega_{\mathrm{FS}}(P)=\sum_{j=2}^{n} d u_{j} \wedge d v_{j}$.

Since $t_{j}=z_{j} / z_{1}$ on $\mathcal{M}$ around $P$, the $z$-coordinates provide a chart of $\mathcal{M}$ around $P$. Calculations show that in these coordinates

$$
\begin{aligned}
\pi^{*} \omega_{\mathrm{std}}+\lambda^{2} q^{*} \omega_{\mathrm{FS}}(P) & =\sum_{j=1}^{n} d x_{j} \wedge d y_{j}+\lambda^{2} \sum_{j=2}^{n} d u_{j} \wedge d v_{j} \\
& =\sum_{j=1}^{n} d x_{j} \wedge d y_{j}+\frac{\lambda^{2}}{c^{2}}\left(\sum_{j=2}^{n} d x_{j} \wedge d y_{j}\right) \\
& =d x_{1} \wedge d y_{1}+\sum_{j=1}^{n} d{\frac{\left(r^{2}+\lambda^{2}\right)}{r}}^{1 / 2} x_{j} \wedge d{\frac{\left(r^{2}+\lambda^{2}\right)}{r}}^{1 / 2} y_{j}(P) \\
& =\left(\pi^{*} h_{\lambda}^{*} \omega_{\mathrm{std}}\right)(P) .
\end{aligned}
$$

Then in $P$ we have that $\rho(1, \lambda)(P)=\pi^{*} h_{\lambda}^{*} \omega_{\text {std }}(P)$. Now since $h_{\lambda}$ and $\pi$ are compatible under the natural $U(n)$-action and $\omega_{\text {std }}$ and $\rho(1, \lambda)$ are invariant under the same action we can deduce that $\pi^{*} h_{\lambda}^{*} \omega_{\text {std }}=\rho(1, \lambda)$ everywhere on $\mathcal{M}$ away from $\pi^{-1}(0)$.

Lemma 4.5 For every monotone embedding $F: \mathbb{C}^{n}-\{0\} \rightarrow \mathbb{C}^{n}$ the form $F^{*} \omega_{\text {std }}$ is Kähler.

Proof Since $F$ is monotone, it is once again compatible with the natural $U(n)$-actions on $\mathbb{C}^{n}$ and $\mathbb{C}^{n}-\{0\}$. Furthermore, since

$$
(d F)_{(F(1), 0, \ldots, 0)}\left(\frac{\partial}{\partial x_{1}}\right)=\frac{d F}{d r}(F(1), 0, \ldots, 0) \cdot\left(\frac{\partial}{\partial x_{1}}\right),
$$

and $\frac{d F}{d r}(F(1), 0, \ldots, 0)>0$ as $F$ is strictly increasing and $\frac{\partial}{\partial x_{j}}$ and $\frac{\partial}{\partial y_{k}}$ are left invariant by $F$ at $(F(1), 0, \ldots, 0)$, for $j=2, \ldots, n$ and $k=1, \ldots, n$, we can deduce that $F^{*} \omega_{\text {std }}(F(1), 0, \ldots, 0)$ is compatible with the complex structure.

Proposition 4.6 ([7, Proposition 5.1A]) For every $\eta, \lambda>0$ there exists a Kähler form $\bar{\tau}=\bar{\tau}(\eta, \lambda)$ on $\mathcal{M}$ such that:

(1) $\bar{\tau}_{\mid \mathcal{M}-\mathcal{M}(1+\eta)}=\pi^{*} \omega_{\text {std }}$.

(2) $\bar{\tau}_{\mid \mathcal{M}(\delta)}=\rho(1, \lambda)$ for some $\delta>0$.

Proof Take $\delta<1+\eta$ such that $h_{\lambda}\left(B_{\delta}^{2 n}(0)\right) \subset B_{\lambda(1+\eta / 2)}^{2 n}(0)$. Then we can use a smoothing procedure to find a monotone embedding $F$, such that:

(1) $F(z)=\lambda z$ for $|z|>1+\eta$.

(2) $F(z)=h_{\lambda}(z)$ for $|z|<\delta$.

The form $\bar{\tau}=\pi^{*}\left(F^{*}\left(\omega_{\text {std }}\right)\right)$ extended to $\pi^{-1}(0)$ has the required properties. 
Construction 4.7 (Symplectic blow-up of a Kähler manifold) Let $(M, \omega)$ be an $n$ dimensional Kähler manifold with a Kähler form $\omega$ and fix a point $P \in M$. Assume that

$$
\phi:\left(B_{(1+2 \eta)}^{2 n}(0), \omega_{\text {std }}\right) \hookrightarrow(M, \omega)
$$

is a holomorphic embedding with $\phi(0)=P$ and $\phi^{*} \omega=\lambda^{2} \omega_{\text {std }}$ for $\lambda \in \mathbb{R}_{>0}$.

Since $\mathcal{M}(1+2 \eta)$ and $B_{1+2 \eta}^{n}(0)$ are holomorphic away from 0 and $\pi^{-1}(0)$ respectively, we can glue in $\mathcal{M}(1+2 \eta)$ instead of $B_{1+2 \eta}^{n}(0)$ and obtain the blown-up complex variety $\tilde{M}$, with the blow-up map $\Pi: \widetilde{M} \rightarrow M$ and exceptional divisor $E=\pi^{-1}(0)$ :

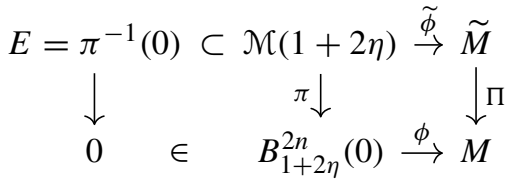

We can construct a Kähler form $\widetilde{\omega}$ on $\tilde{M}$ with $\phi^{*} \widetilde{\omega}=\omega_{\text {std }}$ as follows:

$$
\widetilde{\omega}=\left\{\begin{array}{lll}
\Pi^{*} \omega & \text { on } & \tilde{M}-\widetilde{\phi}(\mathcal{M}(1+2 \eta) \\
\left(\widetilde{\phi}^{-1}\right)^{*} \bar{\tau}(\eta, \lambda) & \text { on } & \widetilde{\phi}(\mathcal{M}(1+2 \eta))
\end{array}\right.
$$

where $\bar{\tau}(\eta, \lambda)$ is defined as above. This is possible since $\widetilde{\phi}^{*} \widetilde{\omega}$ coincides with $\phi^{*} \omega=\omega_{\text {std }}$ on $\mathcal{M}(1+2 \eta)-\mathcal{M}(1)$ by construction of $\tilde{\tau}(\eta, \lambda)$. Moreover $\tilde{\omega}$ is a Kähler form as the two glued components are Kähler forms.

In the setting of Construction 4.7 if $M$ is a projective complex manifold and [ $\widetilde{\omega}]$ the ample class $\left[\pi^{*} \omega\right]-\lambda[E]$ on $M$, where $[E]$ is the cohomology class of (the Poincaré dual of) the exceptional divisor $E$ then $\widetilde{\omega}$ represents an ample class on $\widetilde{M}$. By construction $[\widetilde{\omega}]$ is in the interior of a cone generated by integral Kähler classes. Kodaira's embedding theorem says that Kähler forms representing such integral Kähler classes are curvature forms of Kähler metrics on ample line bundles.

From Construction 4.7 it is clear that to define a symplectic form on the blow-up of a symplectic manifold $M$ at points $P_{1}, \ldots, P_{k}$ of $X$ is equivalent to specifying a holomorphic embedding

$$
\phi: \bigsqcup_{i=1}^{k}\left(B_{i}\left(\lambda_{i}\right), \omega_{\text {std }}\right) \hookrightarrow(M, \omega)
$$

which sends the center of the $i$ th ball to $P_{i}$ for every $i=1, \ldots, k$. Then one can cut out images of the embedded balls and collapse their boundaries to copies of $\mathbb{C P}^{n-1}$ called exceptional divisors. Conversely, given a symplectic form on the blow-up, by blowing down we obtain a symplectic form $\omega$ on $M$ and a packing of $(M, \omega)$ by balls. Both constructions preserve Kähler forms. In terms of the blow-down this amounts 
to determining if the cohomology class on the blow-up of $M$ can be represented by symplectic forms.

\section{Kähler packings of projective, complex, manifolds}

Theorem 5.1 Let $X$ be a projective complex manifold of dimension $n, L$ an ample line bundle on $X$, and $P_{1}, \ldots, P_{k} \in X$. Let $\epsilon_{0}=\epsilon\left(X ; L, P_{1}, \ldots, P_{k}\right)$ denote the multipoint Seshadri constant of $L$ on $X$ in $P_{1}, \ldots, P_{k}$. Then, for any radius $r<\sqrt{\epsilon_{0}}$ there exists a Kähler packing of $k$ flat Kähler balls of radius $r$ into $X$.

Proof First we construct families $\widetilde{X}_{i}$ as in Sect. 3. Then we embed Fubini-Study Kähler balls of large enough volume on $\mathcal{E}_{i}^{(i)}-E_{i}$ provided with the Kähler metric $\omega_{i}^{(i)}$ induced by the global sections of $\widetilde{\mathcal{L}}^{(i)}$ constructed in Theorem 3.4 on $\mathcal{E}_{i}^{(i)}$, for $i=k, k-1, \ldots, 1$. These balls can be deformed to Kähler balls on non-central fibers $\widetilde{X}_{i, \delta_{i}} \cong \widetilde{X}_{i-1}$, and then iteratively to non-central fibers $\widetilde{X}_{j, \delta_{j}} \cong \widetilde{X}_{j-1}$ for $j=i-1, \ldots, 1$ if the $\delta_{j}$ are chosen small enough. Doing this carefully the deformed balls will not intersect on $\widetilde{\widetilde{X}}_{i, \delta_{i}}$ and descend to disjoint balls over $X$. Moreover, there exists a symplectomorphism between flat Kähler balls and Fubini-Study balls given by

$$
\phi:\left(B_{0}^{2 n}(1), \omega_{\mathrm{std}}\right) \hookrightarrow\left(\mathbb{C}^{n}, \omega_{\mathrm{FS}}\right) \subset\left(\mathbb{C P}^{n}, \omega_{\mathrm{FS}}\right)
$$

such that

$$
\left(z_{1}, \ldots, z_{n}\right) \mapsto \frac{1}{\left(1-\sum_{i=1}^{n}\left|z_{i}\right|^{2}\right)^{1 / 2}} \cdot\left(z_{1}, \ldots, z_{n}\right)
$$

This symplectomorphism along with its inverse allows us to glue Fubini-Study balls into flat Kähler balls and vice versa. Hence, gluing standard Kähler balls into the Fubini-Study Kähler balls we obtain the claim.

In more details:

1. Let $\Delta_{\delta} \subset \mathbb{A}_{\mathbb{C}}^{1}$ denote the open disk of radius $\delta$, with affine parameter $t$. Choose local coordinates $y_{1}, \ldots, y_{n}$ of $X$ around $P_{i}$, so $t, y_{1}, \ldots, y_{n}$ are local coordinates around $\left(0, P_{i}\right)$ in $\tilde{X}_{i}$. Then over the open subset $U_{t} \subset \widetilde{X}_{i}$ where these coordinates are defined there is a chart of the blow-up of $X_{i}$ in $\left(P_{i}, 0\right)$ with coordinates $t, z_{1}, \ldots, z_{n}$ such that the blow-up map to $\mathcal{U}_{t}$ is described by $\left(t, z_{1}, \ldots, z_{n}\right) \mapsto\left(t, y_{1}, \ldots, y_{n}\right):=\left(t, t z_{1}, \ldots, t z_{n}\right)$. Because the coordinates $y_{i}$ are bounded, the central fiber of the induced projection of $\mathcal{U}_{t}$ onto $\mathbb{A}_{\mathbb{C}}^{1}$ is $\mathcal{E}_{i}^{(i)}-E \cong \mathbb{A}_{\mathbb{C}}^{n}$. The non-central fibers are not quite isomorphic to $\mathbb{A}_{\mathbb{C}}^{n}$ but contain balls $B_{R}(0)$ with $R$ arbitrarily large close to $t=0$. Thus for $R$ arbitrarily large we can find $\delta$ sufficiently small and an embedding $\iota_{i}: \Delta_{\delta} \times B_{R}(0) \hookrightarrow \widetilde{X}_{i}$. See Fig. 3 .

2. Choosing in $1 . R$ large enough and $\delta$ small enough implies that there exist embeddings of Fubini-Study Kähler balls $B_{R^{\prime}}(0)$ with $R^{\prime} \leqslant R$, of volume arbitrarily 


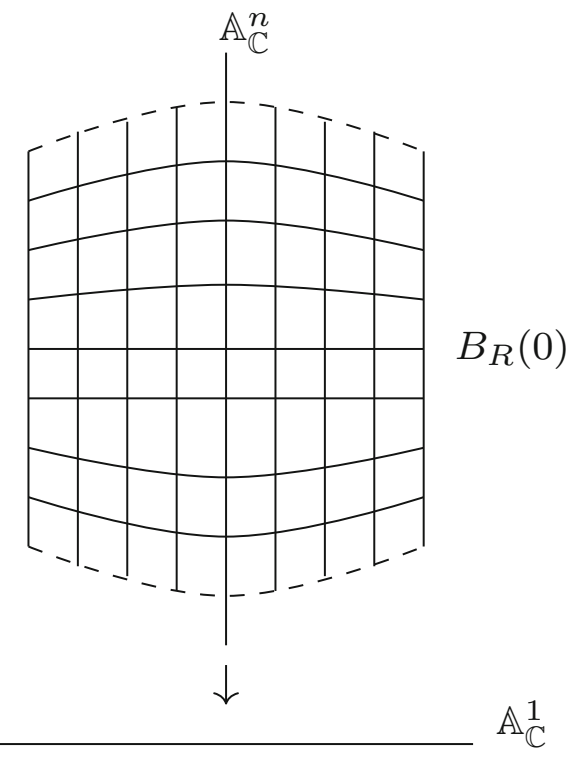

Fig. 3 Illustration of embedded ball away from central fiber.

close to the volume of $\left(\varepsilon_{i}^{(i)}, \omega_{i}^{(i)}\right)$ in all fibers of $\Delta_{\delta} \times B_{R}(0)$ over $t \in \Delta_{\delta}$ with respect to the same Fubini-Study Kähler form $\omega_{i}^{(i)}$.

3. By continuity, for $t$ small enough these Fubini-Study forms $\omega_{i}^{(i)}$ differ to an arbitrarily small amount from the Kähler form $\omega_{i, t}$ on $\widetilde{X}_{i, t}$ obtained from the trivializing sections of $\widetilde{\mathcal{L}}^{(i)}$ pulled back via the embedding $\iota_{i}$. This allows us to glue in the Fubini-Study Kähler balls of step 2. into non-central fibers $\widetilde{X}_{i, t}$ provided with the Kähler form $\omega_{i, t}$ for $t \ll 1$ small enough. Assume that $\omega_{i}^{(i)}=\frac{i}{2 \pi} \partial \bar{\partial} \log \left(s_{i}^{(i)}\right)$ and $\omega_{i, t}=\frac{i}{2 \pi} \partial \bar{\partial} \log s_{i, t}$ on $\{t\} \times B_{R}(0)$, where $s_{i}^{(i)}, s_{i, t}$ are functions constructed in the usual way from the appropriate sections. Then choose a partition of unity $\left(\rho_{1}, \rho_{2}\right)$ such that $\left.\rho_{1}\right|_{B_{R^{\prime}}(0)} \equiv 1$ and $\left.\rho_{2}\right|_{B_{R^{\prime}}(0)} \equiv 0$. The glued 2-form $\widetilde{\omega}_{i, t}$ is given by $\frac{i}{2 \pi} \partial \bar{\partial} \log \left(\rho_{1} s_{i}^{(i)}+\rho_{2} s_{i, t}\right)=\frac{i}{2 \pi} \partial \bar{\partial} \log \left(\rho_{1}\left(s_{i}^{(i)}-s_{i, t}\right)+s_{i, t}\right)$. This form is obviously closed, and it is non-degenerate because $s_{i}^{(i)}-s_{i, t}$ gets arbitrarily small for $t \ll 0$, thus $\rho_{1}\left(s_{i}^{(i)}-s_{i, t}\right)+s_{i, t}$ is arbitrarily close to $s_{i, t}$. Consequently, $\widetilde{\omega}_{i, t}$ is a Kähler form. Furthermore, since the gluing is done on a function/metric level and $\omega_{i, t}$ is obtained by applying $i \partial \bar{\partial} \log$, the gluing factors vanish in the cohomology class. Hence $\left[\widetilde{\omega}_{i, t}\right]=\left[\omega_{i, t}\right]=c_{1}\left(\widetilde{L}^{i-1}\right)$.

4. The $i^{\text {th }}$ Fubini-Study Kähler ball on $\widetilde{X}_{i, \delta_{i}}$ does not intersect the $(i+1)^{\text {st }}, \ldots, k^{\text {th }}$ Kähler ball constructed before: The $i^{\text {th }}$ ball is deformed from a ball on the central fiber $\widetilde{X}_{i, 0}$ lying in $\mathcal{E}_{i}^{(i)}-E_{i}$ whereas the other balls are deformed from balls in $\tilde{X}_{i}-E_{i} \subset \tilde{X}_{i, 0}$. So these balls do not intersect on the central fiber, and this will not change when we deform the balls to $\widetilde{X}_{i, \delta_{i}}$ if we choose $\delta_{i}$ small enough. 
5. Assume $\int \omega_{\mathrm{FS}}^{n}=1$, and $\int_{B_{1}(0)} \omega_{\mathrm{std}}=1$. Then there exists a Kähler embedding $\left(B_{r}(0), \omega_{\mathrm{std}}\right) \hookrightarrow\left(\mathbb{C P}^{n}, \omega_{\mathrm{FS}}\right)$, for all $r<1$. Hence for all $r<\sqrt{m_{i}}$ there exists a Kähler embedding $\left(B_{r}(0), \omega_{\mathrm{std}}\right) \hookrightarrow\left(\mathbb{C P}^{n}, m_{i} \omega_{\mathrm{FS}}\right)$. Rescaling by $d_{i}$ we obtain a Kähler embedding $\left(B_{r}(0), \omega_{\text {std }}\right) \hookrightarrow\left(\mathbb{C P}^{n}, \frac{m_{i}}{d_{i}} \omega_{\mathrm{FS}}\right)$ for all $r<\sqrt{m_{i} / d_{i}}$. Since $\omega_{i}^{(i)}=m_{i} \omega_{\mathrm{FS}}$, the embeddings constructed above imply that $m_{i} / d_{i}<\epsilon_{0}$, but since $m_{i} / d_{i}$ can be chosen arbitrarily close to $\epsilon_{0}$ we can conclude that $\sqrt{\epsilon_{0}} \leqslant \gamma_{k}$, where $\gamma_{k}$ is the $k$-ball packing constant.

Proof of Theorem 1.4 The claim that the $k$-point Seshadri constant is less or equal to the Kähler packing constant is a direct consequence of Theorem 5.1. The converse argument is a consequence of the symplectic blow-up construction detailed in Sect. 4. The embedded balls allow us to construct Kähler forms on the blow-up $\pi$ of the centres whose curvature lies in the first Chern class of $\pi^{*} L-\sum \gamma E_{i}$.

Remark 5.2 The method to prove Theorem 5.1 also allows us to construct Kähler packings of balls with radius $r_{1}, \ldots, r_{k}$ arbitrarily close to $\epsilon_{1}, \ldots, \epsilon_{k}$ as long as $\pi^{*} L-$ $\sum_{i=1}^{k} \epsilon_{i} E_{i}$ is nef.

Acknowledgements The author would like to thank his advisor Thomas Eckl for suggesting this project and providing advice and guidance throughout. He would also like to thank the anonymous referee for suggestions on how to improve the exposition and Kieran Fleming for taking the time to read an early version of this paper and offer suggestions on the formatting and layout.

Open Access This article is licensed under a Creative Commons Attribution 4.0 International License, which permits use, sharing, adaptation, distribution and reproduction in any medium or format, as long as you give appropriate credit to the original author(s) and the source, provide a link to the Creative Commons licence, and indicate if changes were made. The images or other third party material in this article are included in the article's Creative Commons licence, unless indicated otherwise in a credit line to the material. If material is not included in the article's Creative Commons licence and your intended use is not permitted by statutory regulation or exceeds the permitted use, you will need to obtain permission directly from the copyright holder. To view a copy of this licence, visit http://creativecommons.org/licenses/by/4.0/.

\section{References}

1. Bauer, T., Di Rocco, S., Harbourne, B., Kapustka, M., Knutsen, A., Syzdek, W., Szemberg, T.: A primer on Seshadri constants. In: Bates, D.J., et al. (eds.) Interactions of Classical and Numerical Algebraic Geometry. Contemporary Mathematics, vol. 496, pp. 33-70. American Mathematical Society, Providence (2009)

2. Biran, P.: From symplectic packing to algebraic geometry and back. In: Casacuberta, C., et al. (Eds.) European Congress of Mathematics, Vol. II. Progress in Mathematics, vol. 202, pp. 507-524. Birkhäuser, Basel (2001)

3. Demailly, J.-P.: Singular Hermitian metrics on positive line bundles. In: Hulek, K., et al. (eds.) Complex Algebraic Varieties. Lecture Notes in Mathematics, vol. 1507, pp. 87-104. Springer, Berlin (1992)

4. Eckl, T.: Kähler packings and Seshadri constants on projective complex surfaces. Differential Geom. Appl. 52, 51-63 (2017)

5. Hartshorne, R.: Algebraic Geometry. Graduate Texts in Mathematics, vol. 52. Springer, New York (1977)

6. Lazarsfield, R.: Positivity in Algebraic Geometry. Vol. I. Ergebnisse der Mathematik und ihrer Grenzgebiete. 3. Folge, vol. 4. Springer, Berlin (2004)

7. McDuff, D., Polterovich, L.: Symplectic packings and algebraic geometry. With an appendix by Y. Karshon. Invent. Math. 115(3), 405-434 (1994)

8. Trusiani, A.: Multipoint Okounkov bodies (2018). arXiv:1804.02306 
9. Witt Nyström, D.: Okounkov bodies and the Kähler geometry of projective manifolds (2015). arXiv: $1510.00510 \mathrm{v} 3$

Publisher's Note Springer Nature remains neutral with regard to jurisdictional claims in published maps and institutional affiliations. 